\title{
Dormancy in the stem cell niche
}

\author{
Roberta Sottocornola and Cristina Lo Celso*
}

\begin{abstract}
Tissues characterized by constant turnover contain post-mitotic, terminally differentiated cells originating from highly proliferative progenitors, which in turn derive from a relatively small population of stem cells. At the population level, self-renewal and differentiation are the possible outcomes of stem cell proliferation; overall, however, stem cells are quiescent if compared with their direct progeny. The recent discovery of a particularly quiescent, or dormant, subpopulation of hematopoietic stem cells (HSCs) raises a number of fundamental questions. As stem cell fate is influenced by the signals integrated by the stem cell niche, will dormant HSCS reside in specific dormant niches? Is the mechanism of dormancy common to multiple regenerating tissues or specific to the hematopoietic system? If cancer is maintained by a few cancer stem cells, do they also contain a subpopulation of dormant cells, and could this be exploited for therapeutic purposes?
\end{abstract}

\section{Introduction}

Stem cells maintain regenerating tissues such as the epidermis, the intestinal epithelium, and the hematopoietic system thanks to their unique abilities to selfrenew and to generate differentiating progeny. The balance between self-renewal and differentiation is controlled by the interaction between stem cells and the specific microenvironment in which they reside, the 'stem cell niche' [1]. Although stem cells have a high potential to proliferate, they are rather inactive in terms of cell cycle, most likely to prevent their own exhaustion [2] and to protect themselves from acquiring potentially tumorigenic mutations [3,4]. Stem cell quiescence is an evolutionarily conserved mechanism: it was initially demonstrated not only in mammalian epidermis [5] but

\footnotetext{
*Correspondence: clocelso@imperial.ac.uk
}

Department of Life Sciences, Division of Cell and Molecular Biology, Imperial College London, Sir Alexander Fleming Building, South Kensington Campus, London, SW7 2AZ, UK also in the plant root apical meristem [6]. While relative quiescence remains a hallmark of somatic stem cells compared with non-self-renewing progenitors, mathematical modeling of hematopoietic stem cell (HSC) proliferative behavior identified a subpopulation of deeply quiescent mouse HSCs, called dormant HSCs, which divide only five times in the mouse lifetime $[7,8]$. In this article, we review the latest findings on dormant HSCs; we look at epidermis, intestinal epithelium, and neuroepithelium to examine whether the presence of dormant stem cells is a recurring biological mechanism; and we present a few initial examples of new therapeutic developments based on the discovery of dormant stem cells.

\section{Label retention assays measure proliferative behavior}

Slow-dividing somatic stem cells have been identified for many years by using variations of the label retention assay (Figure 1), which is based on in vivo labeling of cell nuclei by means of a pulse of ${ }^{3} \mathrm{H}$-thymidine or nucleotide analogs such as 5-bromo-2'-deoxyuridine (BrdU), followed by a 'chase' period in the absence of a DNAlabeling agent. While dividing cells rapidly dilute the label, long-lived quiescent cells, like stem cells, retain the label over time and can be identified as label-retaining cells (LRCs) [5,9]. In tissues containing other types of long-lived quiescent cells, like the bone marrow, slowdividing stem cells can be identified through their ability to retain the label combined with the expression of specific cell surface markers [10]. One of the main concerns with the use of BrdU to identify LRCs is its inability to be incorporated by non-dividing cells, raising the possibility that the most quiescent cells, which are likely not to undergo any cell division during the pulse phase, may never be labeled. Moreover, BrdU could be detected only on fixed samples and did not allow purification of viable LRCs. The generation of transgenic mice expressing the fusion protein histone 2B-green fluorescent protein (H2B-GFP) under the control of a tetracycline regulatory element provided a new tool to overcome these limitations. These mice were originally developed to identify and purify epidermal LRCs [11] and were crossed with mice harboring the tetracycline-regulated transcription factor TetRVP16 (tTA) under the control of the keratin-5 promoter, leading to double-transgenic 


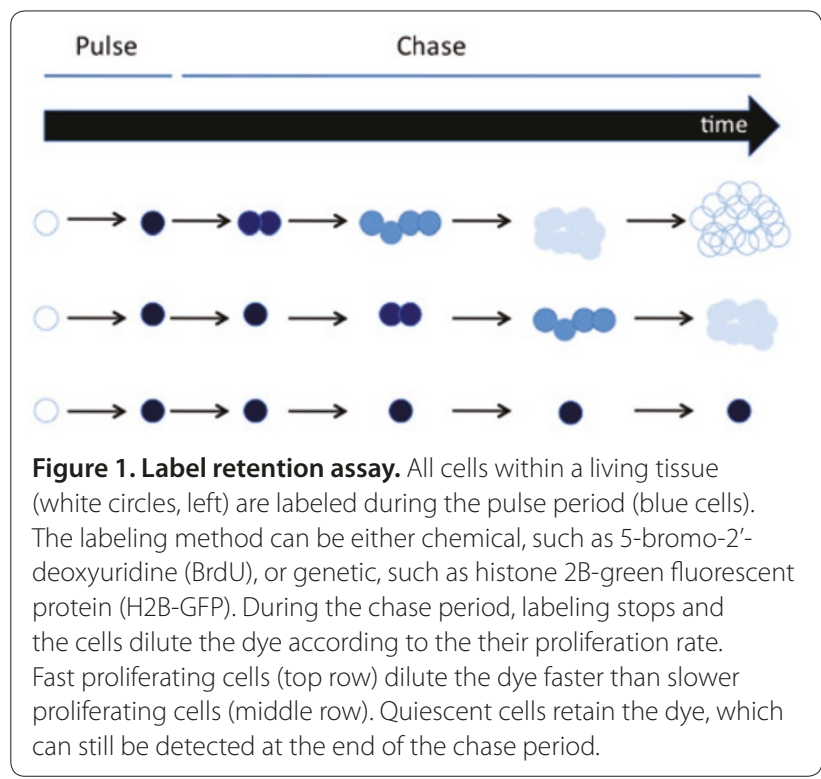

progeny expressing H2B-GFP throughout the basal layer of the epidermis. Since the expression of H2B-GFP is independent from the initial proliferative status of the cell, this system ensures uniform labeling of cells within the tissue. The addition of tetracycline to the diet of the animal inactivates TetRVP16 and switches off the expression of H2B-GFP (chase). During this time, proliferating cells dilute H2B-GFP to undetectable levels within their progeny, whereas quiescent cells are identified as bright $\mathrm{H}_{2} \mathrm{~B}-\mathrm{GFP}^{+}$cells in histological sections and can be isolated alive by using flow cytometry, allowing their further functional characterization.

Alternatively, the reverse form of TetRVP16 transcription factor (rtTA) can be used to express H2B-GFP in the presence of tetracycline, followed by an antibiotic-free chase phase. Several tissue-specific as well as ubiquitous promoters have been used to drive the expression of either tTA or rtTA, and several tissues have been examined for the presence of LRCs and therefore of a potential stem cell reservoir [12-15].

\section{Hematopoietic stem cells and their niche}

HSCs reside in the bone marrow and give rise to erythrocytes, platelets, and all immune cells. Their heterogeneity was first suggested over 50 years ago, when serial transplantation studies showed that fewer than $1 \%$ of spleen colony-forming units (CFU-S) (at the time considered the most refined population of HSCs) possess the capacity to reconstitute lethally irradiated recipient mice [16]. HSCs were subsequently subdivided in long-term reconstituting HSCs (LT-HSCs), which are capable of serially regenerating all blood cell types in transplant recipient mice thanks to their extensive ability to self-renew, and short-term reconstituting HSCs (ST-HSCs), which have a less extensive ability to self-renew. Specific molecular markers have been used to identify and purify LT-HSCs and ST-HSCs [17-20].

Cell cycle analysis revealed that, on average, two thirds of LT-HSCs are in the $\mathrm{G}_{0}$ phase of the cell cycle and that $G_{0}$ LT-HSCs are responsible for the functional engraftment of lethally irradiated recipient mice $[21,22]$. Analysis of the kinetics of BrdU incorporation failed to reveal the existence of a subpopulation of highly quiescent HSCs, as greater than $99 \%$ of LT-HSCs incorporated BrdU after long periods of administration. It was therefore postulated that LT-HSCs regularly enter the cell cycle and divide, on average, every 57 days. Similar conclusions were reached in another study, based on the label retention assay, also using BrdU as a DNA-labeling agent [10].

In a recent study by Wilson and colleagues [8], mice expressing tTA under the control of the stem cell leukemia $(S C L)$ gene promoter were crossed with Tetresponsive $\mathrm{H} 2 \mathrm{~B}-\mathrm{GFP}$ mice, leading to expression of $\mathrm{H} 2 \mathrm{~B}-$ GFP in HSCs and progenitor cells. Mathematical modeling of the results obtained from pulse-chase experiments combined with the use of six different molecular markers phenotypically identifying LT-HSCs $\left(\mathrm{Lin}^{-}, \mathrm{Sca}^{+}, \mathrm{CKit}^{+}\right.$, $\mathrm{CD}_{150^{+}}, \mathrm{CD} 48^{-}$, and $\left.\mathrm{CD} 34^{-}\right)$uncovered the existence of a subpopulation of dormant HSCs, which represented about one seventh of the studied population and which were capable of retaining H2B-GFP label after several months of chase. These HSCs divide every 145 days, which is equivalent to five divisions per average C57/BL6 mouse lifetime. Similar results were obtained by using transgenic mice in which the expression of H2B-GFP is ubiquitously switched on, rather than off, by the addition of doxycycline [7]. In both cases, serial transplantation assays showed that dormant HSCs possess better longterm repopulation potential in comparison with more frequently dividing HSCs. Mobilizing agents such as granulocyte colony-stimulating factor (G-CSF) as well as injury signals, such as 5-fluorouracil or interferon-alpha treatments, and even BrdU administration trigger proliferation in dormant HSCs, suggesting that they act as a reservoir of stem cells in emergency situations rather than contribute to steady-state hematopoiesis $[8,23]$.

HSC niches are specified by the combination of several molecular signals that are produced by different cell types in different locations within the bone marrow and that control stem cell fate [24,25]. Although dormant HSCs are likely to rely on specific signals from their niche, no specific cell type has been unequivocally associated with dormancy so far. Osteoblasts are known to produce several factors able to induce HSC quiescence and therefore, potentially, dormancy [26-28]. It has been suggested that $\mathrm{N}$-cadherin-positive, spindle-shaped osteoblasts might directly support HSC quiescence [25]. Wilson and 
colleagues [8] found c-Kit ${ }^{+}$, BrdU LRCs near endosteal surfaces in the bone sections of mice after 170 days of chase, suggesting that the endosteum could harbor dormant HSC niches. However, a recent report indicated that endothelium-derived angiopoietin-like 3 also induces HSC quiescence, raising the question of whether multiple stroma cell types could be responsible for induction of HSC quiescence and dormancy [29].

\section{Hair follicle stem cell niche}

The epidermis is a multilayered epithelium comprising the interfollicular epidermis and annexed structures such as hair follicles and sebaceous and sweat glands. Hair follicles regenerate themselves through cycles of growth (anagen), destruction (catagen), and rest (telogen), suggesting the presence of an intrinsic stem cell population. Although epidermal stem cells exist in the interfollicular epidermis and sebaceous glands [30], the best-characterized population of epidermal stem cells resides in the hair follicle bulge [31]. Situated just below the sebaceous glands, the bulge marks the lower limit of the permanent region of the hair follicles and contains slow-cycling LRCs $[11,31,32]$. Bulge cells not only contribute to hair follicle homeostasis as shown by lineage-tracing experiments [11,33] but also can proliferate in response to injury stimuli such as wounding and phorbol ester [34].

The identification of a subpopulation of bulge cells with long-term label-retaining properties $[34,35]$ suggests that these infrequently dividing cells may represent the longterm stem cell population of the tissue. Two recent studies from the Tumbar group [36,37] identified slowdividing hair follicle stem cells but ruled out this hypothesis by following the proliferation of bulge LRCs during the first and second hair follicle cycles. Although a fraction of slow-dividing cells (around 28\% of total bulge cells) were present, all bulge cells divided within two subsequent hair cycles, and the majority of cells divided three times, on average, during one hair cycle. Given that hair follicles undergo approximately 20 hair cycles in the lifetime of the mouse, it can be estimated that bulge cells divide fewer than 100 times. While these observations seem to exclude the existence of a deeply quiescent population of hair follicle stem cells comparable to LRCHSCs, it is likely that the epidermal stem cell pool harbors further complexity. Genetic lineage-tracing experiments in adult mice have shown that bulge cells repair the wound only transiently, raising the possibility that long-term stem cells involved in tissue repair, like dormant HSCs, may be present in the epidermis [35].

As hypothesized for the HSC niche, in the hair follicle active and quiescent stem cells are found in distinct locations. The bulge region is known to produce inhibitory signals and therefore favors stem cell quiescence [11], whereas the dermal papilla induces activation of stem cells in the hair germ [38]. In vivo tracking experiments have shown that labeled, quiescent bulge cells abandon the niche prior to embarking on proliferation and differentiation [37], suggesting the existence of quiescent and active subniches within the bulge itself.

\section{Intestinal stem cell niche}

The intestinal epithelium, which covers the surface of the intestinal lumen, provides a remarkable example of highly regenerating tissue, and so the debate is open about the existence of dormant intestinal stem cells (ISCs). Epithelial sheets renew quickly under constant insults such as exposure to digestive enzymes and mechanical erosion. This constant regeneration starts in the intestinal crypts, which harbor a population of multipotent ISCs. The identification of the precise localization of ISCs in the crypts has been a challenging task for researchers over the years because of the lack of specific markers to distinguish ISCs from other progenitors. A seminal study by Potten and colleagues [39] identified a population of cells that were capable of retaining ${ }^{3} \mathrm{H}$-thymidine or BrdU over several weeks and that were localized at the base of the crypts around the +4 position, which has been widely assumed to represent the stem cell niche in the small intestine. Although providing the basis for future development in the characterization of ISCs, this study did not grant any information on the potency or regenerating ability of these LRCs.

Functional ISCs were identified by using the molecular marker Lgr5, a gene coding for a G protein-coupled receptor, expressed below the +4 position at the base of the crypts [27]. Mice expressing a tamoxifen-inducible Cre knock-in allele into the $\operatorname{Lgr} 5$ locus were crossed with the Cre-activatable R26R-LacZ reporter strain to perform lineage-tracing experiments marking the progeny of $\mathrm{Lgr}^{+}$cells. Sixty days after tamoxifen injection, entire crypts were positive for LacZ, indicating that $\mathrm{LgrS}^{+}$cells are capable of generating all intestinal lineages. More recently, similar experiments identified Bmi1, a chromatin remodeling protein of the polycomb family, as a marker of ISCs at the +4 position of intestinal crypts [40]. Because in this study the labeling of the entire crypt was achieved only 12 months after tamoxifen induction, it can be inferred that $\mathrm{BmI}^{+}$and $\mathrm{Lgr5^{+ }}$ cells generate progeny with different kinetics. More recently, mouse telomerase reverse transcriptase was shown to mark relatively quiescent, crypt-regenerating cells located in various positions near the bottom of the intestinal crypt [41]. The presence of stem cells with different proliferation profiles in distinct locations of the crypt points to the existence of a complex array of signaling molecules that are produced in different places within the same niche and that are able to control cell fate through 
inhibition or promotion of quiescence. Interestingly, highly quiescent stem cells that are able to proliferate in response to injury while retaining their potential were identified in the Drosophila hindgut [42]. These observations raise the possibility that, similarly to the hematopoietic system and the hair follicles, different populations of stem cells with different cycling kinetics could coexist, possibly within the same niche, and be responsible for homeostatic and injury-triggered regeneration. The combination of newly identified molecular markers for ISCs with the H2B-GFP-labeling strategy could be the next step in investigating the existence of a population of dormant stem cells in the mammalian small intestine.

\section{Neuroepithelium of the adult mammalian brain}

The subgranular zone of the hippocampal dentate gyrus (DG) represents one of the main reservoirs of neural stem cells (NSCs) in the adult mammalian brain. NSCs in this region of the brain are characterized by the expression of the transcription factor Sox2 and divide infrequently. In transgenic mice harboring GFP under the control of the Sox 2 promoter, fewer than $10 \%$ of the $\mathrm{GFP}^{+}$ cells colocalize with cell proliferation markers [43]. A recent study suggests that bone morphogenetic protein (BMP) signaling is an important switch between the quiescent and the proliferative status of these cells [44]. Administration of the BMP antagonist Noggin to mice treated with BrdU increased the proliferation of Sox $2^{+}$ cells in the hippocampus by recruiting quiescent cells into the cell cycle [44]. Analysis of the DG in mice expressing GFP under the control of Hes5, one of the main targets of Notch signaling, suggested that the vast majority of Sox ${ }^{+}$cells with both radial and horizontal morphology also express Hes5 [45]. Similarly to what was reported for Sox2 transgenics, the administration of BrdU to these mice allowed the labeling of only a small fraction of NSCs, further supporting the notion that they are highly quiescent. When BrdU was administered for 15 days followed by a chase period of 30 days, only a few $\mathrm{GFP}^{+} \mathrm{BrdU}^{+}$cells were found in the DG and some of them were positive for PCNA (proliferating cell nuclear antigen), indicating that NSCs shuttle between a mitotically active and a quiescent state. Interestingly, different stimuli triggered the activation of different subsets of $\mathrm{GFP}^{+}$cells; physical activity recruited radial quiescent cells into the cell cycle, whereas seizure induced by the administration of kainic acid resulted in the proliferation of horizontal $\mathrm{GFP}^{+}$cells. Although the existence of quiescent NSCs in the DG has been well documented, further experiments are still needed to determine their kinetics of cell division and establish whether these quiescent cells are in a dormant state similar to the one described for HSCs.

\section{Dormancy and cancer stem cells: therapeutic implications}

As cancer stem cells (CSCs) are able to give rise to the bulk of the tumor, they share some characteristic traits of normal stem cells. Thus, CSCs represent the population with tumor propagation potential within the tumor itself. The resistance of CSCs to radiotherapy and chemotherapy has been proposed to account for relapse and metastasis formation after initial successful treatments. Several mechanisms, such as relatively efficient DNA repair and expression of transporter pumps able to expel drugs out of the cell, have been proposed to explain such resistance [46]. Recent studies led to the hypothesis that deep quiescence could protect LSCs from the effect of anti-proliferative agents. For example, non-cycling AML stem cells have been shown to reside in the bone marrow endosteal region [47], and cultured CD34 ${ }^{+}$chronic myeloid leukemia (CML) stem cells isolated from patients with CML contain a fraction of highly quiescent cells resistant to imatinib mesylate (IM), a tyrosine kinase inhibitor used for the treatment of CML [48].

The similarities between LSCs and HSCs and the association between dormancy and residence in the niche led to a number of studies exploring the use of G-CSF to activate and mobilize LSCs in order to sensitize them to chemotherapy. Indeed, the exposure of CD34+ CML stem cells to G-CSF before IM treatment resulted in activation of quiescent cells, enhancing the effects of IM on these otherwise-resistant cells [48]. Treatment with G-CSF of a xenograft model, generated by transplanting purified LSCs from patients with acute myeloid leukemia (AML) in immunocompromised mice, resulted in the activation of AML stem cells. Interestingly, these activated cells could be partially eliminated by treatment with the chemotherapeutic agent cytarabine [47]. Even though the mice in this study could not be completely cured of leukemia, these data suggest that activation of LSCs combined with chemotherapy could represent the first step toward a successful treatment of the disease. Pandolfi and colleagues [49] recently showed that combined treatment of a CML mouse model with $\mathrm{As}_{2} \mathrm{O}_{3}$ and Ara- $\mathrm{C}$ resulted in the complete eradication of the disease. $\mathrm{As}_{2} \mathrm{O}_{3}$, which targets the tumor suppressor PML for degradation, inhibits quiescence of LSCs and therefore sensitizes them to the pro-apoptotic effect of the chemotherapeutic drug Ara-C.

As CSCs have also been identified in several solid tumors, approaches similar to those developed for leukemia treatment could lead to better eradication of several types of tumors in addition to hematological malignancies. Inactivation of the oncogene MYC was associated with tumor dormancy in a model of hepatocellular carcinoma [50], suggesting that targeting of specific molecules in solid tumors could also represent a powerful therapeutic intervention to break the dormancy of CSCs. 


\section{Conclusions}

The availability of a number of specific cell surface markers allowed the isolation, purification, and functional characterization of HSCs in vitro and in vivo, leading to the recent identification of a population of highly quiescent, injury-responsive, dormant HSCs. As the full nature of the HSC niche remains elusive, the challenge now is to understand whether dormant and homeostatic HSCs segregate in specific niches or coexist in subsections of the same niche. The comparison of the hematopoietic system with other tissues characterized by high turnover, such as the epidermis and the intestinal epithelium, leads to an emerging pattern of allocation of duties between somatic stem cell subpopulations, with some of them being responsible for day-to-day maintenance and others being set aside for prompt repair of injury, and a similar pattern is emerging even for organs characterized by very slow turnover, such as the brain. While histological analysis and whole-mount preparations provide excellent tools for performing detailed label retention and lineage-tracing analysis in the epidermis and the intestine $[42,45]$, it is likely that further developments in three-dimensional live imaging technology will be needed in order to generate a clear picture of the localization and behavior of dormant and homeostatic somatic stem cells [51]. The combination of the different experimental approaches currently used for each tissue will likely solve the debate on the prescence of dormant stem cells and their niches.

The discovery of dormant stem cells brings the new challenge of understanding the molecular signals controlling maintenance of, exit from, and return to dormancy. If dormant stem cells are indeed able to respond to injury and revert to dormancy unaltered, harnessing their potential will provide insights on how to prevent aging and how to enhance regeneration following disease. At the same time, a better understanding of the molecular regulation of CSC quiescence is starting to provide new avenues for the development of improved therapeutic interventions.

This article is part of a review series on Stem cell niche.

Other articles in the series can be found online at

http://stemcellres.com/series/stemcellniche

\begin{abstract}
Abbreviations
AML, acute myeloid leukemia; BMP, bone morphogenetic protein; BrdU, 5-bromo-2'-deoxyuridine; CML, chronic myeloid leukemia; CSC, cancer stem cell; DG, dentate gyrus; G-CSF, granulocyte colony-stimulating factor; GFP, green fluorescent protein; H2B-GFP, histone 2B-green fluorescent protein; HSC, hematopoietic stem cell; IM, imatinib mesylate; ISC, intestinal stem cell; LRC, label-retaining cell; LT-HSC, long-term reconstituting hematopoietic stem cell; NSC, neural stem cell; rtTA, reverse form of TetRVP16 transcription factor; ST-HSC, short-term reconstituting hematopoietic stem cell; tTA, tetracyclineregulated transcription factor TetRVP16.
\end{abstract}

\section{Competing interests}

The authors declare that they have no competing interests.

\section{Acknowledgments}

We are grateful to Soline Estrach and Kim Jensen for critical input on the manuscript and to Nahema Rajbahli for initial bibliographic and graphic contributions. The work of RS is funded by Cancer Research UK (CRUK). $\mathrm{CLC}$ receives funding from CRUK, the Kay Kendall Leukaemia Fund, the Biotechnology and Biological Sciences Research Council, and the Human Frontier Science Program.

Published: 19 March 2012

\section{References}

1. Schofield R: The relationship between the spleen colony-forming cell and the haemopoietic stem cell. Blood Cells 1978, 4:7-25.

2. Orford KW, Scadden DT: Deconstructing stem cell self-renewal: genetic insights into cell-cycle regulation. Nat Rev Genet 2008, 9:115-128.

3. Park Y, Gerson SL: DNA repair defects in stem cell function and aging. Annu Rev Med 2005, 56:495-508

4. Wang JC, Dick JE: Cancer stem cells: lessons from leukemia. Trends Cell Biol 2005, 15:494-501.

5. Bickenbach JR: Identification and behavior of label-retaining cells in oral mucosa and skin. J Dent Res 1981, 60 Spec No C:1611-1620.

6. Doerner P: Root development: quiescent center not so mute after all. Curr Biol 1998, 8:R42-44

7. Foudi A, Hochedlinger K, Van Buren D, Schindler JW, Jaenisch R, Carey V, Hock $\mathrm{H}$ : Analysis of histone 2B-GFP retention reveals slowly cycling hematopoietic stem cells. Nat Biotechno/ 2009, 27:84-90.

8. Wilson A, Laurenti E, Oser G, van der Wath RC, Blanco-Bose W, Jaworski M, Offner S, Dunant CF, Eshkind L, Bockamp E, Lio P, Macdonald HR, Trumpp A: Hematopoietic stem cells reversibly switch from dormancy to self-renewal during homeostasis and repair. Cell 2008, 135:1118-1129.

9. Chwalinski S, Potten CS, Evans G: Double labelling with bromodeoxyuridine and $[3 \mathrm{H}]$-thymidine of proliferative cells in small intestinal epithelium in steady state and after irradiation. Cell Tissue Kinet 1988, 21:317-329.

10. Kiel MJ, He S, Ashkenazi R, Gentry SN, Teta M, Kushner JA, Jackson TL, Morrison SJ: Haematopoietic stem cells do not asymmetrically segregate chromosomes or retain BrdU. Nature 2007, 449:238-242.

11. Tumbar T, Guasch G, Greco V, Blanpain C, Lowry WE, Rendl M, Fuchs E: Defining the epithelial stem cell niche in skin. Science 2004, 303:359-363.

12. Brennand $K$, Huangfu D, Melton D: All beta cells contribute equally to islet growth and maintenance. PLOS Bio/ 2007, 5:e163.

13. Oliver JA, Maarouf O, Cheema FH, Martens TP, Al-Awqati Q: The renal papilla is a niche for adult kidney stem cells. J Clin Invest 2004, 114:795-804.

14. Szotek PP, Chang HL, Brennand K, Fujino A, Pieretti-Vanmarcke R, Lo Celso C, Dombkowski D, Preffer F, Cohen KS, Teixeira J, Donahoe PK: Normal ovarian surface epithelial label-retaining cells exhibit stem/progenitor cell characteristics. Proc Natl Acad Sci U S A 2008, 105:12469-12473.

15. Tsujimura A, Koikawa Y, Salm S, Takao T, Coetzee S, Moscatelli D, Shapiro E, Lepor H, Sun TT, Wilson EL: Proximal location of mouse prostate epithelial stem cells: a model of prostatic homeostasis. J Cell Bio/ 2002, 157:1257-1265.

16. Till JE, McCulloch EA: A direct measurement of the radiation sensitivity of normal mouse bone marrow cells. Radiat Res 1961, 14:213-222.

17. Adolfsson J, Borge OJ, Bryder D, Theilgaard-Monch K, Astrand-Grundstrom I, Sitnicka E, Sasaki Y, Jacobsen SE: Upregulation of Flt3 expression within the bone marrow Lin(-)Sca1(+)c-kit(+) stem cell compartment is accompanied by loss of self-renewal capacity. Immunity 2001, 15:659-669.

18. Bryder D, Rossi DJ, Weissman IL: Hematopoietic stem cells: the paradigmatic tissue-specific stem cell. Am J Pathol 2006, 169:338-346.

19. Christensen JL, Weissman IL: Flk-2 is a marker in hematopoietic stem cell differentiation: a simple method to isolate long-term stem cells. Proc Natl Acad SciU SA 2001, 98:14541-14546.

20. Osawa M, Hanada K, Hamada H, Nakauchi H: Long-term lymphohematopoietic reconstitution by a single CD34-low/negative hematopoietic stem cell. Science 1996, 273:242-245.

21. Cheshier SH, Morrison SJ, Liao X, Weissman IL: In vivo proliferation and cell cycle kinetics of long-term self-renewing hematopoietic stem cells. Proc Natl Acad Sci U S A 1999, 96:3120-3125.

22. Passegue E, Wagers AJ, Giuriato $S$, Anderson WC, Weissman IL: Global 
analysis of proliferation and cell cycle gene expression in the regulation of hematopoietic stem and progenitor cell fates. J Exp Med 2005,

202:1599-1611.

23. Essers MA, Offner S, Blanco-Bose WE, Waibler Z, Kalinke U, Duchosal MA, Trumpp A: IFNalpha activates dormant haematopoietic stem cells in vivo. Nature 2009, 458:904-908.

24. Calvi LM, Adams GB, Weibrecht KW, Weber JM, Olson DP, Knight MC, Martin RP, Schipani E, Divieti P, Bringhurst FR, Milner LA, Kronenberg HM, Scadden DT: Osteoblastic cells regulate the haematopoietic stem cell niche. Nature 2003, 425:841-846

25. Zhang J, Niu C, Ye L, Huang H, He X, Tong WG, Ross J, Haug J, Johnson T, Feng JQ, Harris S, Wiedemann LM, Mishina Y, Li L: Identification of the haematopoietic stem cell niche and control of the niche size. Nature 2003, 425:836-841.

26. Arai F, Hirao A, Ohmura M, Sato H, Matsuoka S, Takubo K, Ito K, Koh GY, Suda T: Tie2/angiopoietin-1 signaling regulates hematopoietic stem cell quiescence in the bone marrow niche. Cell 2004, 118:149-161.

27. Barker N, van Es JH, Kuipers J, Kujala P, van den Born M, Cozijnsen M, Haegebarth A, Korving J, Begthel H, Peters PJ, Clevers H: Identification of stem cells in small intestine and colon by marker gene Lgr5. Nature 2007, 449:1003-1007.

28. Yoshihara H, Arai F, Hosokawa K, Hagiwara T, Takubo K, Nakamura Y, Gomei Y, I wasaki H, Matsuoka S, Miyamoto K, Miyazaki H, Takahashi T, Suda T: Thrombopoietin/MPL signaling regulates hematopoietic stem cell quiescence and interaction with the osteoblastic niche. Cell Stem Cell 2007, 1:685-697.

29. Zheng J, Huynh H, Umikawa M, Silvany R, Zhang CC: Angiopoietin-like protein 3 supports the activity of hematopoietic stem cells in the bone marrow niche. Blood 2011, 117:470-479.

30. Ghazizadeh S, Taichman LB: Multiple classes of stem cells in cutaneous epithelium: a lineage analysis of adult mouse skin. EMBO J 2001, 20:1215-1222

31. Cotsarelis G, Sun TT, Lavker RM: Label-retaining cells reside in the bulge area of pilosebaceous unit: implications for follicular stem cells, hair cycle, and skin carcinogenesis. Cell 1990, 61:1329-1337.

32. Morris RJ, Potten CS: Highly persistent label-retaining cells in the hair follicles of mice and their fate following induction of anagen. J Invest Dermatol 1999, 112:470-475.

33. Taylor G, Lehrer MS, Jensen PJ, Sun TT, Lavker RM: Involvement of follicular stem cells in forming not only the follicle but also the epidermis. Cell 2000, 102:451-461.

34. Braun KM, Watt FM: Epidermal label-retaining cells: background and recent applications. J Investig Dermatol Symp Proc 2004, 9:196-201.

35. Ito M, Yang Z, Andl T, Cui C, Kim N, Millar SE, Cotsarelis G: Wnt-dependent de novo hair follicle regeneration in adult mouse skin after wounding. Nature 2007, 447:316-320.

36. Waghmare SK, Bansal R, Lee J, Zhang YV, McDermitt DJ, Tumbar T: Quantitative proliferation dynamics and random chromosome segregation of hair follicle stem cells. EMBO J 2008, 27:1309-1320.

37. Zhang YV, Cheong J, Ciapurin N, McDermitt DJ, Tumbar T: Distinct selfrenewal and differentiation phases in the niche of infrequently dividing hair follicle stem cells. Cell Stem Cell 2009, 5:267-278.

38. Blanpain C, Lowry WE, Geoghegan A, Polak L, Fuchs E: Self-renewal, multipotency, and the existence of two cell populations within an epithelial stem cell niche. Cell 2004, 118:635-648.
39. Potten CS, Owen G, Booth D: Intestinal stem cells protect their genome by selective segregation of template DNA strands. J Cell Sci 2002, 115:2381-2388.

40. Sangiorgi $\mathrm{E}$, Capecchi MR: Bmi1 is expressed in vivo in intestinal stem cells. Nat Genet 2008, 40:915-920.

41. Montgomery RK, Carlone DL, Richmond CA, Farilla L, Kranendonk ME, Henderson DE, Baffour-Awuah NY, Ambruzs DM, Fogli LK, Algra S, Breault DT: Mouse telomerase reverse transcriptase (mTert) expression marks slowly cycling intestinal stem cells. Proc Natl Acad Sci U S A 2011, 108:179-184.

42. Fox DT, Spradling AC: The Drosophila hindgut lacks constitutively active adult stem cells but proliferates in response to tissue damage. Cell Stem Cell 2009, 5:290-297.

43. Suh H, Consiglio A, Ray J, Sawai T, D'Amour KA, Gage FH: In vivo fate analysis reveals the multipotent and self-renewal capacities of Sox $2+$ neural stem cells in the adult hippocampus. Cell Stem Cell 2007, 1:515-528.

44. Mira H, Andreu Z, Suh H, Lie DC, Jessberger S, Consiglio A, San Emeterio J, Hortiguela R, Marques-Torrejon MA, Nakashima K, Colak D, Gotz M, Farinas I, Gage FH: Signaling through BMPR-IA regulates quiescence and long-term activity of neural stem cells in the adult hippocampus. Cell Stem Cell 2010, 7:78-89.

45. Lugert S, Basak O, Knuckles P, Haussler U, Fabel K, Gotz M, Haas CA, Kempermann G, Taylor V, Giachino C: Quiescent and active hippocampal neural stem cells with distinct morphologies respond selectively to physiological and pathological stimuli and aging. Cell Stem Cell 2010, 6:445-456

46. Zhou BB, Zhang H, Damelin M, Geles KG, Grindley JC, Dirks PB: Tumourinitiating cells: challenges and opportunities for anticancer drug discovery. Nat Rev Drug Discov 2009, 8:806-823.

47. Saito Y, Uchida N, Tanaka S, Suzuki N, Tomizawa-Murasawa M, Sone A, Najima Y, Takagi S, Aoki Y, Wake A, Taniguchi S, Shultz LD, Ishikawa F: Induction of cell cycle entry eliminates human leukemia stem cells in a mouse model of AML. Nat Biotechnol 2010, 28:275-280.

48. Jorgensen HG, Copland M, Allan EK, Jiang X, Eaves A, Eaves C, Holyoake TL: Intermittent exposure of primitive quiescent chronic myeloid leukemia cells to granulocyte-colony stimulating factor in vitro promotes their elimination by imatinib mesylate. Clin Cancer Res 2006, 12:626-633.

49. Ito K, Bernardi R, Morotti A, Matsuoka S, Saglio G, Ikeda Y, Rosenblatt J, Avigan DE, Teruya-Feldstein J, Pandolfi PP: PML targeting eradicates quiescent leukaemia-initiating cells. Nature 2008, 453:1072-1078.

50. Shachaf CM, Kopelman AM, Arvanitis C, Karlsson A, Beer S, Mandl S, Bachmann MH, Borowsky AD, Ruebner B, Cardiff RD, Yang Q, Bishop JM, Contag $\mathrm{CH}$, Felsher DW: MYC inactivation uncovers pluripotent differentiation and tumour dormancy in hepatocellular cancer. Nature 2004, 431:1112-1117.

51. Lo Celso C, Lin CP, Scadden DT: In vivo imaging of transplanted hematopoietic stem and progenitor cells in mouse calvarium bone marrow. Nat Protoc 2011, 6:1-14.

doi:10.1186/scrt10

Cite this article as: Sottocornola R, Lo Celso C: Dormancy in the stem cell niche. Stem Cell Research \& Therapy 2012, 3:10. 